OPEN ACCESS

Edited by:

Milica S. Prostran

University of Belgrade, Serbia

Reviewed by:

Fiona Kumfor,

Neuroscience Research Australia,

Australia

Francesca Morganti,

University of Bergamo, Italy

*Correspondence: Ambra Bisio ambra.bisio@gmail.com

Received: 16 November 2015 Accepted: 22 February 2016 Published: 07 March 2016

Citation:

Bisio A, Casteran M, Ballay Y, Manckoundia P, Mourey F and Pozzo T (2016) Voluntary Imitation in

Alzheimer's Disease Patients.

Front. Aging Neurosci. 8:48. doi: 10.3389/fnagi.2016.00048

\section{Voluntary Imitation in Alzheimer's Disease Patients}

\author{
Ambra Bisio ${ }^{1,2 *}$, Matthieu Casteran ${ }^{3}$, Yves Ballay ${ }^{3}$, Patrick Manckoundia ${ }^{3,4}$, \\ France Mourey ${ }^{3}$ and Thierry Pozzo 1,3,5
}

${ }^{1}$ Department of Robotics, Brain and Cognitive Sciences, Istituto Italiano di Tecnologia, Genoa, Italy, ${ }^{2}$ Department of Experimental Medicine, University of Genoa, Genoa, Italy, ${ }^{3}$ INSERM U1093 Cognition, Action et Plasticité Sensorimotrice, Université de Bourgogne Franche-Comté, Dijon, France, ${ }^{4}$ Service de Médecine Interne Gériatrie, Hôpital de Champmaillot, Centre Hospitalier Universitaire, Dijon, France, ${ }^{5}$ Institut Universitaire de France, Université de Bourgogne Franche-Comté, Dijon, France

Although Alzheimer's disease (AD) primarily manifests as cognitive deficits, the implicit sensorimotor processes that underlie social interactions, such as automatic imitation, seem to be preserved in mild and moderate stages of the disease, as is the ability to communicate with other persons. Nevertheless, when AD patients face more challenging tasks, which do not rely on automatic processes but on explicit voluntary mechanisms and require the patient to pay attention to external events, the cognitive deficits resulting from the disease might negatively affect patients' behavior. The aim of the present study was to investigate whether voluntary motor imitation, i.e., a volitional mechanism that involves observing another person's action and translating this perception into one's own action, was affected in patients with AD. Further, we tested whether this ability was modulated by the nature of the observed stimulus by comparing the ability to reproduce the kinematic features of a human demonstrator with that of a computerizedstimulus. AD patients showed an intact ability to reproduce the velocity of the observed movements, particularly when the stimulus was a human agent. This result suggests that high-level cognitive processes involved in voluntary imitation might be preserved in mild and moderate stages of $A D$ and that voluntary imitation abilities might benefit from the implicit interpersonal communication established between the patient and the human demonstrator.

Keywords: motor imitation, action observation, movement execution, Alzheimer's disease, social interaction

\section{INTRODUCTION}

In mild and moderate stages of Alzheimer's disease (AD), despite cognitive decline, some basic mechanisms, such as motor resonance-i.e., automatic activation, during actions perception, of the perceiver's motor system (Rizzolatti et al., 1999), which are believed to underlie natural and spontaneous interaction among humans (Chartrand and Bargh, 1999)-seem to be preserved. In a recent article we showed that the observation of an abstract moving stimulus influenced the motor responses of AD patients (Bisio et al., 2012) suggesting the preservation of motor resonance mechanisms, expressed in the form of automatic imitation,

Abbreviations: AD, Alzheimer's disease; CG, Control group; D, Dot; H, Human demonstrator; F, Fast; M, Medium; S, Slow; RT, Reaction Time; $V_{P}$, Participants' velocity; $V_{H}$, Human demonstrator's velocity; $V_{D}$, Dot velocity. 
which is the involuntary tendency of humans to copy the features of the observed actions (Heyes, 2011). However, when AD patients face more challenging tasks, which do not rely only on automatic processes but also on explicit voluntary mechanisms and require the patient to pay attention to the external events, the cognitive deficits resulting from the disease might negatively affect patient's behavior. This might be the case for voluntary imitation, a social-cognitive mechanism (Korman et al., 2015), which involves observing another person's action and translating explicitly these precepts into one's own actions (Brass and Heyes, 2005).

Voluntary motor imitation makes it possible to interact with others by volitionally sharing behavioral states. It is a powerful biological resource for cognitive development (Meltzoff and Moore, 1977), social interaction (Chartrand and Bargh, 1999) and motor learning (Byrne and Russon, 1998). Altered imitation processes might result in abnormal behavior, as in the case of compulsive imitation behavior (Lhermitte et al., 1986; Pandey and Sarma, 2015). Another pathological condition related to imitation mechanisms is apraxia, defined as the difficulty to produce gestures on verbal command or by imitation. These are examples of the non-memory cognitive symptoms of AD. Several studies that characterized the relationship between disease progression and the different kinds of apraxia generated contrasting results (Edwards et al., 1991; Travniczek-Marterer et al., 1993). While some researchers reported more difficulties to execute transitive gestures (Rapcsak et al., 1989), others showed that AD patients were mostly impaired in the imitation of pantomimes and meaningless (intransitive) movements, the latter explained as being caused by impaired visual-spatial analysis (Rousseaux et al., 2012).

The ability to voluntarily imitate the actions of others could thus be negatively affected by visual deficiencies since it requires intense visuospatial processing and the ability to match the model's posture and movements with one's own motor repertoire (Goldenberg, 1999). Furthermore, it is known that $\mathrm{AD}$ patients exhibit attention deficits, which particularly affect their ability to focus on stimulus modifications and to follow elementary instructions (Perry and Hodges, 1999), as well as that to imitate a specific feature of the observed motion. Another source of difficulty in voluntary imitation could be the kind of stimulus AD patients have to reproduce. Indeed, $\mathrm{AD}$ is also a source of visual dysfunction, such as color discrimination (Cronin-Golomb et al., 1993) and shape recognition, especially when objects or persons are moving (Rizzo and Nawrot, 1998). Consequently, one can expect the imitation of movement features to be impaired in the presence of an enriched stimulus, as in the case of a real person moving in front of the patient, as compared with a simple digitalized visual display. Nevertheless, when $\mathrm{AD}$ patients watch actions performed by a human being, socioemotional processes (Narme et al., 2013) might intervene to improve their ability to relate to and maybe to voluntarily imitate the observed movement's kinematic features. Indeed, it has been shown that the ability to identify social and emotional signals and to access social knowledge is intact in patients with mild and moderate stages of $\mathrm{AD}$, but impaired in other forms of dementia (Shany-Ur and Rankin, 2011).

In the present study we asked AD patients and healthy agematched participants to voluntarily reproduce the kinematic features of two moving stimuli. Precisely, subjects were required to observe and to reproduce the velocity of an abstract, computergenerated stimulus (a dot moving upwards on a screen) and of a human demonstrator, who was facing the participants and performed upward pointing movements with his right arm. In view of the aforementioned considerations, one could expect either the loss or the preservation of $\mathrm{AD}$ patients' capacity to reproduce the velocity of the observed stimuli. In the latter case, the preserved motor resonance could have enabled the voluntary imitation. Furthermore, comparing the imitation performance of $\mathrm{AD}$ patients when they looked at a simple dot with that when they looked at a human demonstrator would shed light on their ability to select and reproduce a precise kinematic schema of the observed motion, even in the case of an enriched stimulus as a human being.

\section{MATERIALS AND METHODS}

\section{Participants}

The experimental group was composed of 23 elderly participants (14 women), from 75 to 86 years of age (mean age, $82 \pm 5$ ), with mild or moderate $\mathrm{AD}$ diagnosed according to the French National Institute of Neurology and Communication Disorders and Strokes-The Alzheimer's Disease and related Disorders Association (NINCDS-ADRDA) and the Diagnostic and Statistical manual-IV-Text Revised (DSM IV-TR) criteria, and living at home or in a nursing home specializing in AD. They did not differ according to their residence. They all underwent a comprehensive diagnostic evaluation, which included a clinical assessment, detailed neuropsychology tests, brain magnetic resonance imaging (MRI) and an examination of motor competencies. All of them presented progressive cognitive impairment, predominantly affecting memory and no evident problem in motor performances. Their Mini-Mental State Examination (MMSE) scores were between 12 and 23 (mean, $17 \pm 4$ ). The control group (CG) was composed of 14 healthy participants (10 women and 4 men), from 75 to 87 years of age (mean age, $82 \pm 4$ ), living at home. Their MMSE scores were between 25 and 30 (mean, $29.5 \pm 1.5$ ). All participants were right-handed, and had normal or corrected-to-normal vision. They were able to hear adequately, to pay attention to the examiner's behavior and to understand elementary questions. Written informed consent was obtained from each participant or their guardians, and the Local Ethics Committee of Burgundy Hospitals (Dijon University Hospital-CHU-CMRR-France) approved the protocol. Table 1 contains the demographic data.

\section{Materials and Procedure}

The experiment lasted about $20 \mathrm{~min}$. A moving stimulus was used as a template to test the effect of motion perception on the execution of subsequent pointing movements. Participants received verbal feedback during the testing procedure in order to 
TABLE 1 | Demographic data of the Alzheimer's disease (AD) patients and healthy age-matched participants (control group, CG).

\begin{tabular}{lccc}
\hline & AD & CG & Statistics \\
\hline Number of subjects & 23 (14 females) & 14 (10 females) & not significant \\
Age (mean years \pm SD) & $82 \pm 5$ & $82 \pm 4$ & not significant \\
Range & $75 \div 86$ & $75 \div 87$ & \\
MMSE (mean \pm SD) & $17 \pm 4$ & $29.5 \pm 1.5$ & $p<<0.01$ \\
Range & $12 \div 23$ & $25 \div 30$ & \\
Education (mean years \pm SD) & $5.07 \pm 1.82$ & $5.21 \pm 1.63$ & not significant \\
Range & $3 \div 8$ & $3 \div 8$ & \\
& & &
\end{tabular}

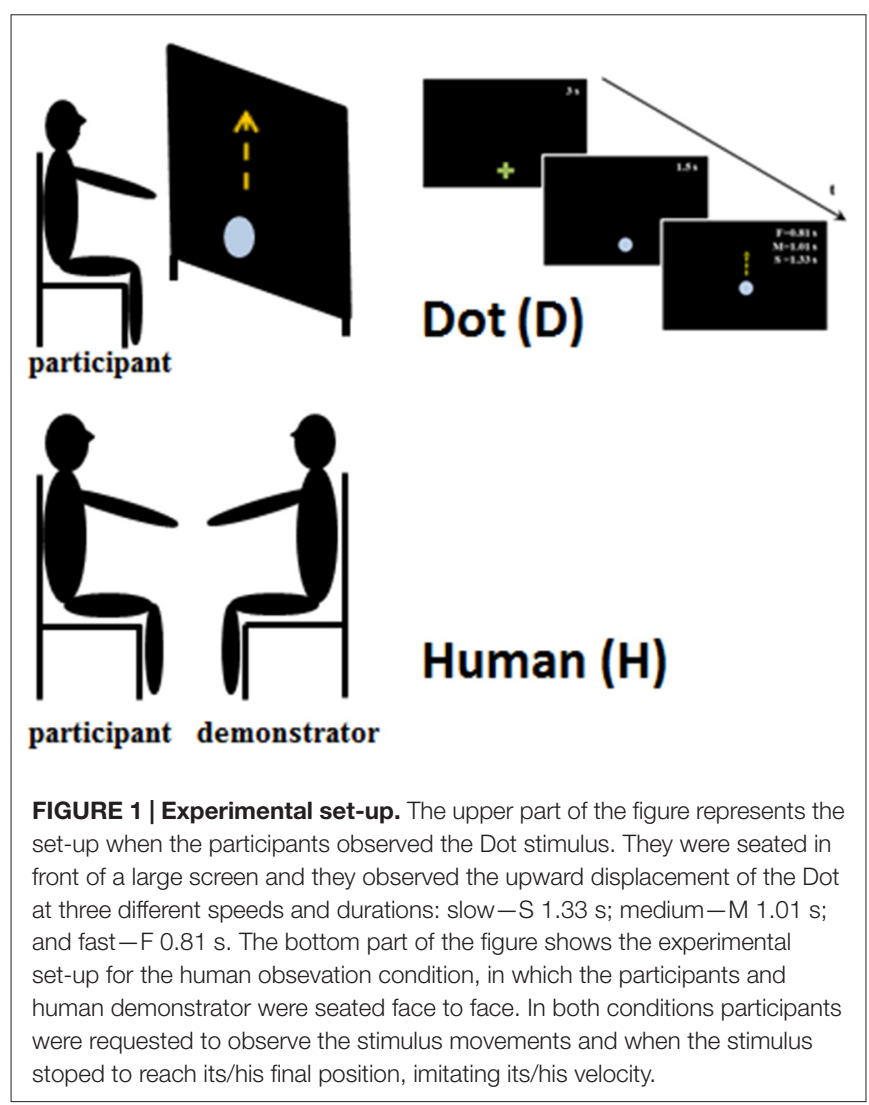

remind them about the experimental instructions. The beginning of each phase was preceded by a training step, which ended when the participant understood the task. The experimental set up is represented in Figure 1.

\section{Dot Observation (D)}

The experiment was performed in a darkened room. Participants sat on a chair, in front of a large rear projection screen $(170 \times 230$ $\mathrm{cm}$ ) at a viewing distance of about $60 \mathrm{~cm}$. A video-projector, with a refresh rate of $60 \mathrm{~Hz}$, placed behind the screen and connected to a PC, back-projected the visual stimuli onto the display screen. The visual stimulus was generated using MatLab Psychtoolbox 3 (Brainard, 1997). An optoelectronic system (SMART) with five cameras was used to record movements at a sampling frequency of $120 \mathrm{~Hz}$. One passive infrared reflective marker was applied onto the tip of the participant's right index finger.
A green cross was displayed at the movement starting position. After $3 \mathrm{~s}$, the green cross was replaced by a light blue dot $(3.2 \mathrm{~cm}$ in diameter). The dot was displayed in this position for $1.5 \mathrm{~s}$, and then started to move vertically, over a distance of $52 \mathrm{~cm}$. Dot motion reproduced the kinematic characteristics of a human vertical movement. The dot moved at three different mean velocities: slow $(0.39 \mathrm{~m} / \mathrm{s}$, corresponding to $1.33 \mathrm{~s})$, medium $(0.51 \mathrm{~m} / \mathrm{s}$, corresponding to a total time of $1.01 \mathrm{~s})$, and fast $(0.64 \mathrm{~m} / \mathrm{s}$, corresponding to $0.81 \mathrm{~s})$. Stimulus velocities were randomized. Participants were asked to point at the green cross, then to watch the movement of the dot until it reached its final (visible) position. When the dot stopped, the participants replicated its movement. We asked the participants to wait until the stimulus stopped to start moving because in such a way they could better appreciate the kinematic feature of the observed movement. Particular emphasis was given to the imitation of the dot velocity. The test was repeated four times for each dot velocity (12 trials in total; Figure 1, upper part).

\section{Human Observation $(\mathbf{H})$}

The person that acted as the stimulus (human demonstrator, $\mathrm{H}$ ) was a young man and was the same in all the experiments. He was previously trained to make vertical straight movements at three different velocities $\left(\mathrm{V}_{\mathrm{H}}\right.$, Slow, Medium and Fast) with his right arm kept in a comfortable position. As in the case of the dot, the human demonstrator's velocities were randomized. The demonstrator and the participant sat on comfortable chairs facing each other. The participants were instructed to point their right index finger at the demonstrator's fingertip, then to watch the movement until it stopped. They then replicated the movement, to reach the demonstrator's final fingertip position. Also in this condition particular emphasis was given to the imitation of the demonstrator's movement velocity. Each participant accomplished 12 movements.

\section{Data Treatment}

\section{Data Processing}

Kinematic data were low-pass filtered at $5 \mathrm{~Hz}$ using a 2nd order Butterworth filter. To define the onset and offset of the movement, we chose a threshold corresponding to $5 \%$ of the maximum value of the movement velocity profile.

\section{Data Analysis}

In order to provide a quantitative description of both the planning and execution phases of participants' movement, reaction time (RT) and mean velocity were considered the outcome variables for all trials.

RT was computed as the difference in time between the end of the stimulus motion and the start of the participant's pointing movement. In order to check participants' ability to suppress the motor response until the stimulus stopped, the percentage of RT $>0$ for each participant after the observation of both $\mathrm{D}$ and $\mathrm{H}$ was calculated. These values were statistically evaluated using a mixed-design analysis of variance (ANOVA) 
with Group (2 levels, CG and AD), as the between-subject factor, and Stimulus (2 levels, D and H), as the within-subject factor.

Participants' $V_{P}$ values were compared using a mixed-model analysis of variance with Group (2 levels, CG and AD) as the between-subject factor, and velocity as the within-subject factor (3 levels, Slow, Medium and Fast). This statistical analysis method was chosen for its flexibility to designs that are not perfectly balanced, as in our case. Moreover, it allowed us to take into account the intrinsic (and uncontrolled) variability among the participants, which was considered everywhere as a random factor. Because of the differences between the dot (D) and the demonstrator's $(\mathrm{H})$ velocities, two distinctive mixed-model analyses of variance were performed on D and $\mathrm{H}$ datasets. In the $\mathrm{H}$ condition, the data were classified on the basis of demonstrator's movement velocity $\left(\mathrm{V}_{\mathrm{H}}\right)$ : i.e., Slow $\mathrm{V}_{\mathrm{H}}<0.4$, Medium $0.4<\mathrm{V}_{\mathrm{H}}<0.7$ and Fast $\mathrm{V}_{\mathrm{H}}>0.7$.

A linear regression model illustrated the relationship between $\mathrm{V}_{\mathrm{D}, \mathrm{H}}$ and $\mathrm{V}_{\mathrm{P}}$ for each participant. The slope of the linear fits was mainly used to evaluate whether and how much participants were able to replicate the stimulus' velocity and can be considered a measure of the accuracy of the imitation. Slope $=1$ indicated the perfect reproduction of the stimulus velocity. A mixed-designed ANOVA, with Group as the betweensubjects factor, and Stimulus (2 levels, $\mathrm{D}$ and $\mathrm{H}$ ), as the with-in subjects factor, was applied on slope values. Significant interactions were always interpreted with Newman-Keuls post hoc comparisons.

Finally, we computed another parameter, which provided information related to participants' imitation ability. This parameter was obtained for each subject by subtracting the slope value of the linear regression model applied to describe the relationship between participant's and stimuli velocities from the slope of the perfect imitation line and calculating the absolute value of this difference $(a b s(1-$ slope $))$; the greater the difference the poorer the imitation performance. Pearson's correlation was applied to assess any correlation between the imitation abilities defined by this index and participants' cognitive status, assessed by the MMSE score.

\section{RESULTS}

All participants completed the experiment. According to an informal interview made at the end of the experiment, none had problems seeing the visual stimuli and none considered the task difficult.

Figure 2 shows the mean percentage of participants' responses that started after the end of stimulus motion (i.e., $\mathrm{RT}>0$ ) as requested by the experimenter. For both dot stimulus and human demonstrator, most healthy participants (CG) were able to wait until the end of the stimulus movement before starting to move (around $80 \%$ of positive RT values). In contrast, most of the $\mathrm{AD}$ patients' responses were anticipated $(\mathrm{RT}<0)$ : i.e., they started to move while the stimulus was still moving. Moreover, when the stimulus was the human demonstrator both groups were better able to follow the instructions than when the stimulus was the dot. These observations were confirmed by

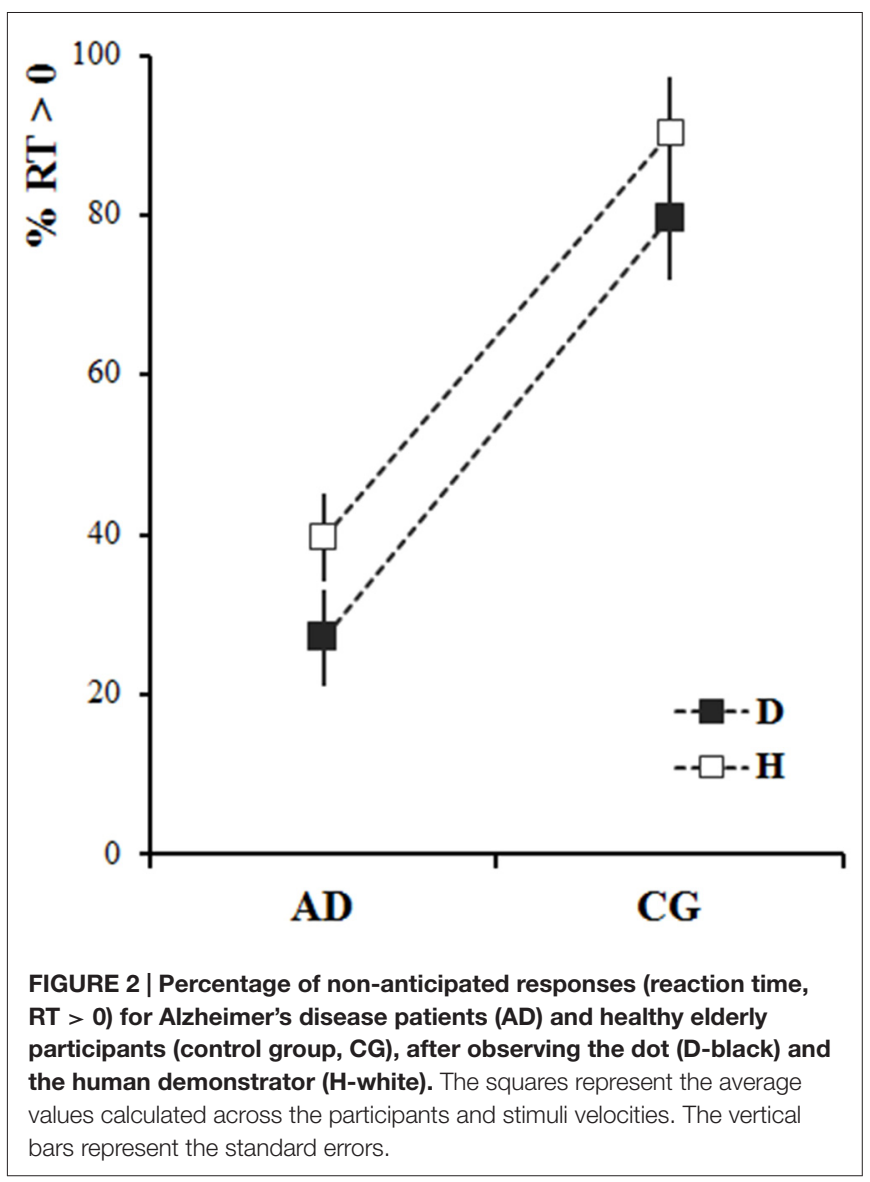

the statistical analysis. The results of the mixed-design ANOVA showed a significant Group effect (the percentage of anticipated responses in $\mathrm{AD}$ patients was significantly higher than that in the $C G-F_{(1,36)}=47.19, p<0.001$ ) and Stimulus effect (the percentage of anticipated responses was lower in both groups when they observed the human demonstrator $-F_{(1,36)}=6.31$, $p<0.05)$.

The mean pointing velocities of participants in the CG and $\mathrm{AD}$ patients $\left(\mathrm{V}_{\mathrm{P}}\right.$, black and white dots, respectively) are represented in Figure 3 as a function of the stimuli velocities $\left(V_{D}\right.$, Figure $3 \mathbf{A}$ and $V_{H}$, Figure $\left.3 \mathbf{B}\right)$. The participants of both groups understood the experimental instructions concerning the imitation of the stimuli velocities. Participants' velocities changed in accordance with the dot and demonstrator's velocities. However, this effect was less pronounced for $\mathrm{AD}$ patients when they observed the dot. Indeed, AD patients' movement velocities were closer to stimulus velocities when the stimulus was a human demonstrator than when it was a dot.

A significant Group*Velocity interaction emerged in data associated with observation of the $\operatorname{dot}\left(F_{(2,71.72)}=17.05\right.$, $p<0.001)$. The subsequent post hoc comparison showed that AD patients' mean velocities for Fast and Slow stimuli differed significantly $(p<0.001)$. Further, the mean velocities of the CG at each stimulus velocity were significantly different one from the other $(p<0.001)$. In addition, the movements performed by the 


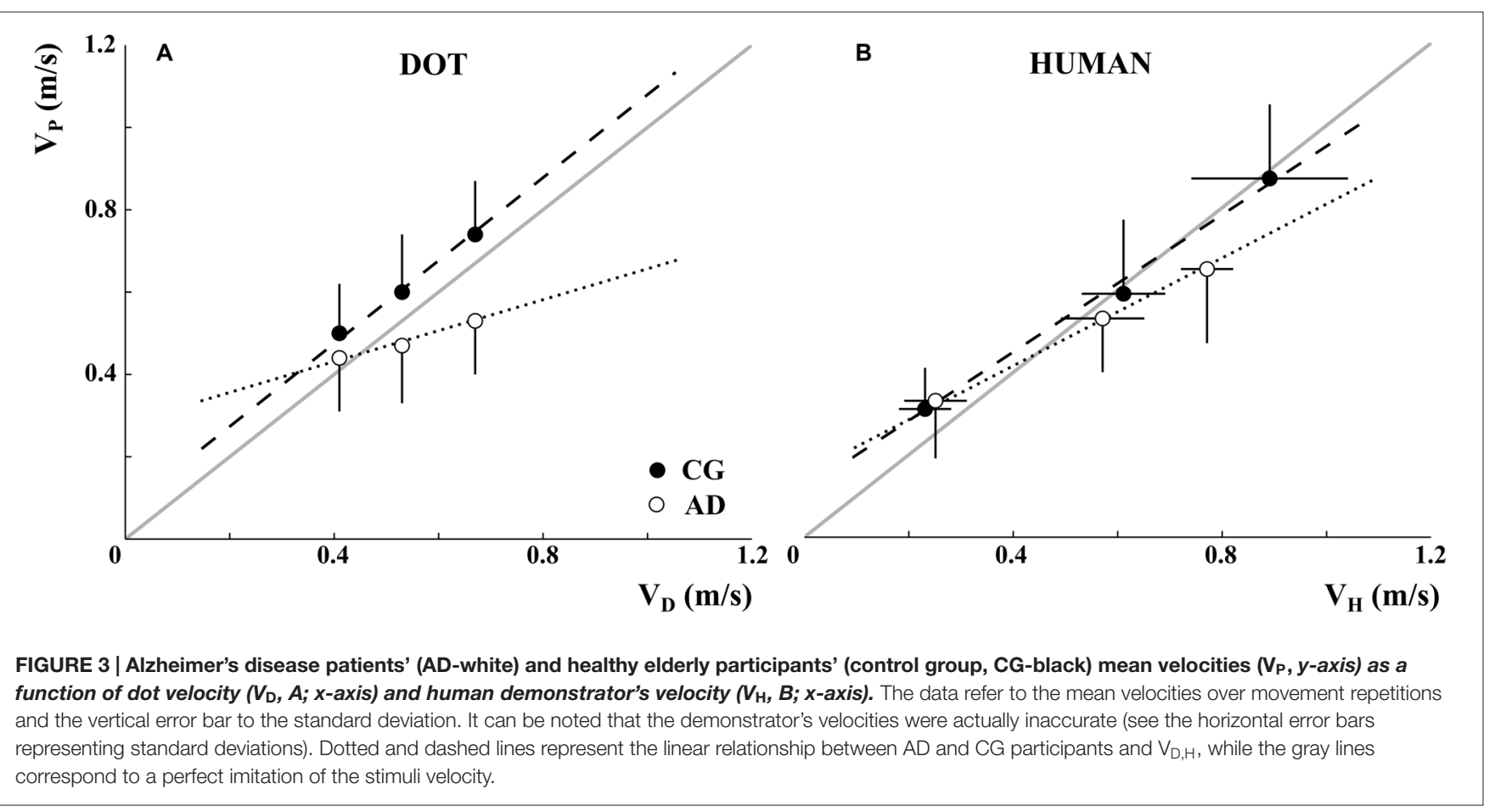

participants in the CG in the Medium and Fast conditions were significantly faster than $\mathrm{AD}$ patients' movements $(p<0.001)$.

The statistical analysis of the data recorded during the human demonstrator condition showed a combined effect of Group and Velocity (Group*Velocity: $F_{(2,51.35)}=3.88, p<0.05$ ). Post hoc comparison showed that for both groups the velocity in Fast condition was higher than in Medium and Slow conditions, and that in the Medium condition was higher than in Slow (always $p<0.001$ ). Moreover, a significant difference between patients' and participants' mean velocities appeared with regard to Fast and Medium stimuli $(p<0.001)$; this result was probably related to the gap between the human demonstrator's velocities in Fast conditions observed by the two groups (see Figure 3B).

ANOVA comparing the slope values (i.e., index of the accuracy of the imitation) in the four experimental conditions showed a significant Group*Stimulus interaction $\left(F_{(1,35)}=14.36\right.$, $p=0.0006$; Figure 4). Concerning the results of the post doc analysis, slope values for the CG were significantly higher when participants observed the dot than when they observed the human demonstrator $(p=0.002)$. The mean slope value for the dot stimulus slightly exceeded $1\left(\right.$ slope $\left._{\mathrm{D}}=1.13\right)$, whereas it was lower than 1 for the human demonstrator $\left(\right.$ slope $_{\mathrm{H}}=0.83$ ). However, if we consider the absolute difference between the perfect imitation and the slope values in the two cases there was only a marginal divergence from 1 (D: 0.13, H:0.17), which cannot be interpreted as a significant difference in the imitation performance. Concerning $\mathrm{AD}$ patients, slope values were significantly higher with the human demonstrator than with the dot stimulus $(p=0.045)$. In addition, whereas AD patients' slope values were significantly lower those of the CG

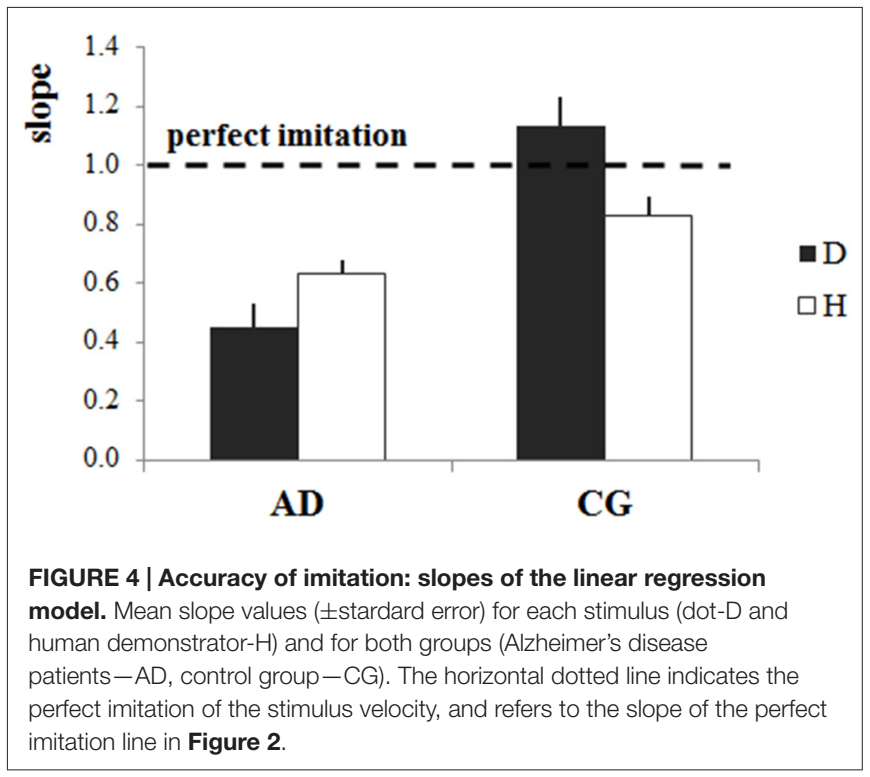

for the dot stimulus $(p=0.0001)$, there were no differences between the slopes values of the two groups for the human demonstrator $(p=0.12)$, meaning that $\mathrm{AD}$ patients imitation performance reached that of the CG when there was a human demonstrator.

Correlation analysis between the imitation index defined as abs(1-slope) and MMSE showed that the more severe the cognitive impairement (low MMSE) the worse the imitation abilities (high values of $a b s(1-$ slope $)$ ). Whereas this result was only a trend for the dot stimulus $(R=-0.28, p=0.09$; Figure 5A), there was a significant negative correlation between the imitation 


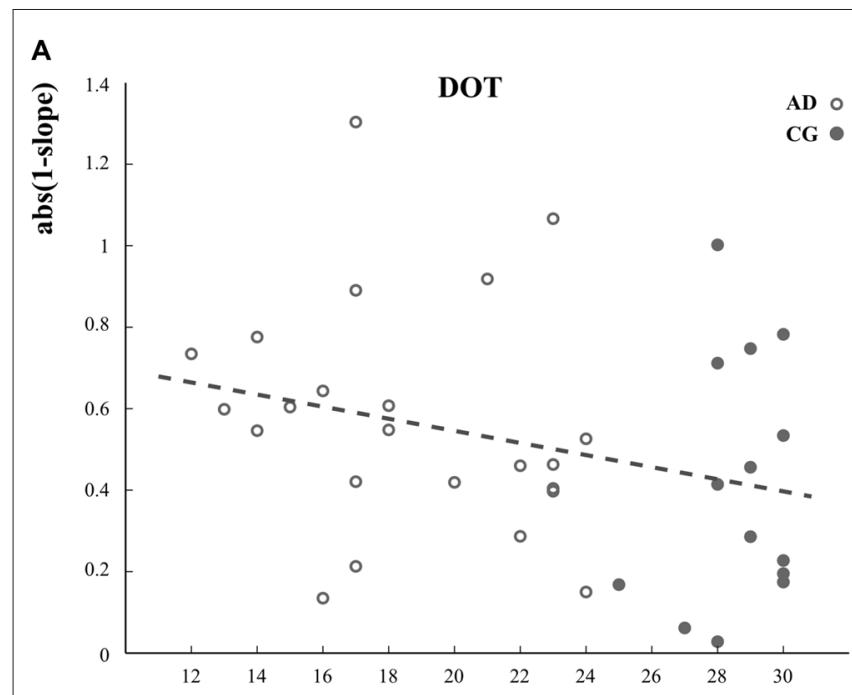

B

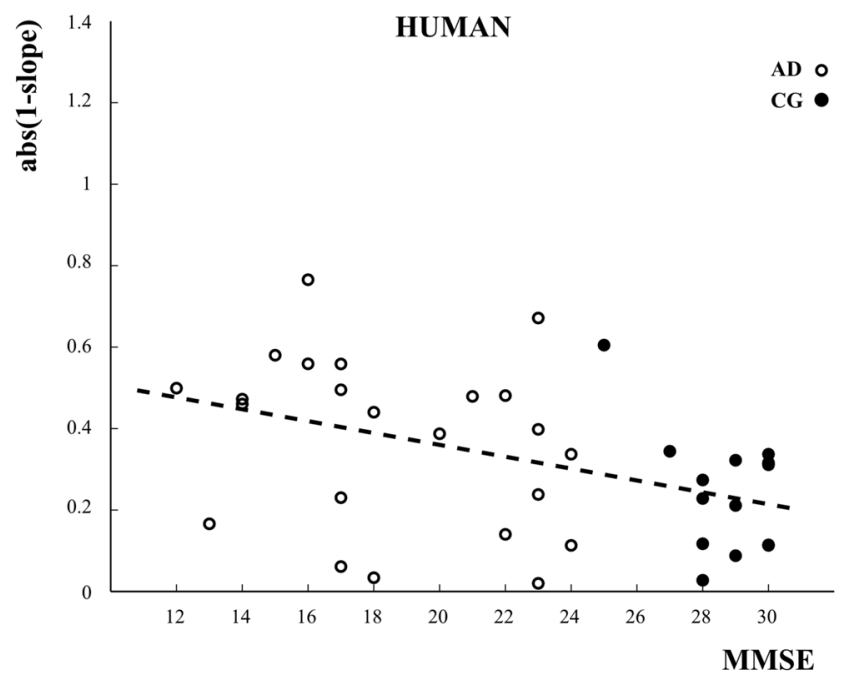

FIGURE 5 | Linear relationships between the imitation index and the cognitive status assessed using the mini mental state examination (MMSE) scores for the dot condition (A, gray circles) and the human demonstrator condition (B, black circles). Open and closed circles refer to the data of $A D$ patients and the control participants (CG), respectively. The imitation index is here defined as the absolute difference between the slope of the perfect imitation line $($ slope $=1$ ) and the slope of the linear regression model applied to describe the relationship between participants' and stimulus velocities. The greater the difference, the worse the imitation performances.

abilities and MMSE when participants oberved the human demonstrator $(R=-0.42, p=0.009$; Figure 5B).

\section{DISCUSSION}

This study aimed to determine AD patients' ability to imitate the kinematic features of two kinds of stimuli: an abstract, computergenerated display (a dot) projected on a large screen, and a human demonstrator facing the participant. The main findings were that: (1) $\mathrm{AD}$ patients were able to imitate the kinematic features of the stimuli; and (2) the imitation performance was better when they observed the human demonstrator than when the stimulus was abstract. Furthermore, the imitation performance worsened with increasing cognitive impairment. Finally, the percentage of anticipated motor responses with respect to the end of the stimulus movement was lower when the stimulus was a human demonstrator.

\section{The Nature of the Stimulus Modulated the Start of the Motor Response}

The present data showed that most healthy participants were able to follow the instruction given by the experimenter about the starting time (i.e., to wait until the stimulus stopped before starting the movement) whereas most $\mathrm{AD}$ patients started to move before the end of stimulus motion. This result confirms our previous work which showed that in a context of implicit automatic imitation, AD patients were not only influenced by the velocity of the observed stimulus, but were compulsively attracted by the display and could not refrain from moving (Bisio et al., 2012). This indicates that the mere presence of a moving stimulus was sufficient to trigger the start of movement in $\mathrm{AD}$ patients. As previously suggested (Bisio et al., 2012), the inability to prevent movement when observing a moving stimulus could reflect inadequate functioning of the inhibitory mechanisms. This dysfunction could be due to altered corticocortical connections linking both the basal forebrain system and the parietal lobes to the frontal lobes (Lhermitte et al., 1986; Aron et al., 2003).

Intriguingly, in both groups, the percentage of nonanticipated responses (i.e., RT $>0$ ) increased when participants looked at the human demonstrator. This effect might be explained by the hypothesis that, compared to a simple dot moving on a screen, a human demonstrator is a more salient and meaningful stimulus in the eyes of the participants. Therefore, by delaying movement onset so as to catch the details of his motion, they could better fulfil the task.

\section{The Interaction with a Human Demonstrator Boosted Alzheimer Patients' Imitation Performance}

Both healthy elderly participants and $\mathrm{AD}$ patients complied with the experimental instruction concerning the imitation of the stimulus velocity: indeed, the velocity of participants' pointing movements increased with increasing stimulus velocity (both $\mathrm{D}$ and $\mathrm{H}$ ). Thus, participants were able to imitate both an abstract computer-generated stimulus and a human demonstrator. Since deficits in motor planning (Edwards et al., 1991; Travniczek-Marterer et al., 1993), attention mechanisms (Perry and Hodges, 1999), visuomotor integration (Gilmore et al., 1994; Rizzo and Nawrot, 1998; Tippett and Sergio, 2006; Tippett et al., 2007), and movement control (Ghilardi et al., 1999) have been reported in individuals with AD-type dementia, this finding was not easily predictable. Our result implies that $\mathrm{AD}$ patients understood the experimental instructions, extracted the correct information from the stimulus (i.e., position and velocity), used that information to plan their motor response, and executed the movement imitating the stimulus velocity. 
Therefore, the high-level cognitive processes that underlie all of these functions seem to be preserved in the mild and moderate phases of $\mathrm{AD}$ and could be exploited during training programs and cognitive rehabilitative interventions. This result also suggests that the kinematic representation of action (e.g., velocity, duration and spatial trajectory) is preserved during pathological (AD) aging. More speculatively, the preserved ability to match the kinematic features of the visual model with the internal motor repertoire could have driven the initial planning phase of $\mathrm{AD}$ motor response, and helped participants to voluntarily replicate the kinematics of the model.

Interestingly, participants' voluntary imitation abilities varied depending on whether they observed the dot or the human demonstrator. The ability of CG participants to reproduce the observed kinematics was not stimulus dependent. This finding is in agreement with those reported in our previous studies (Bisio et al., 2010, 2012, 2014) and could reflect the participants' ability to extract low-level features of motion (i.e., kinematic details of the stimulus) without being influenced by other features of the stimulus not related to the movement itself.

AD patients' behavior contrasted with that of CG participants. Remarkably, although AD individuals varied their movement velocity when velocity of the abstract stimulus changed, the imitation performance improved when they observed a human demonstrator. One hypothesis to explain this effect is that the observation of human movement not only induced an automatic match between the observed movement kinematics and the patient's internal motor repertoire, but also triggered emotional mechanisms associated with social interaction. Generally, in its initial and moderate stages, $\mathrm{AD}$ is dominated by cognitive symptoms, whereas social and emotional functioning is relatively spared (Rosen et al., 2004; Mendez et al., 2005; Rankin et al., 2008) and patients do not exhibit increased levels of interpersonal dysfunction (Rankin et al., 2003). Furthermore, it has been suggested that while $\mathrm{AD}$ may impair cognitive processes, the capacity for emotional communication is preserved (Rankin et al., 2008; Shany-Ur and Rankin, 2011). Faces, bodies, and particularly dynamic body stimuli, carry precious information on the actions, intentions and emotional states of others (de Gelder et al., 2014). Therefore, the perception of this kind of information might have helped patients to accomplish the task, thus leading to a better imitation performance compared with that achieved during dot observation. More speculatively, according to the theories of embodied cognition, which link the cognitive processing of an event to its sensory and motor components, the emotional content of the interaction between $\mathrm{AD}$ patients and the human demonstrator might have enhanced their imitation abilities (Vallet, 2015).

From a neurophysiological point of view, the automatic imitation of the observed movement features suggests that the activity of a circuit which involves the mirror neuron system, the superior temporal sulcus (Iacoboni et al., 1999) and the sensorimotor areas for movement production is preserved. Preservation of the ability to voluntarily imitate movement, as tested here, entails the functioning of a broader neural network that also includes sub-cortical regions, such as the basal ganglia, for the decision making component. Nevertheless, the occurrence of compensatory mechanisms where the intact brain regions take over the functions of the injured areas cannot be ruled out (Buckner, 2004; Nithianantharajah and Hannan, 2009). Unfortunately, we are not able to provide a complete description of the results of the neuroimaging and neuropsychological investigations the patients underwent. Therefore, these interpretations remain speculative.

\section{The Cognitive Status Affected Imitation Performance}

We found that the cognitive status influenced participants' imitation performance. Indeed, the existence of a negative correlation between the voluntary imitation abilities and participants' MMSE shows that increasing cognitive impairment reduces imitation ability. This could reflect a limited comprehension of the experimental instructions in the moderate stage of the pathology. In this case one may have expected a similar result for the two stimuli. However, we found that the relationship became significant only when observing the demonstrator, suggesting more than a difficulty to understand the experimental instruction. Indeed, it is likely that the decrease in the MMSE score was also related to progression of the AD in other fields. Decreasing visual accuracy, less motivation to interact with others, or degradation of the neural networks responsible for imitation are some potential candidates among several others accounting for this result. Future studies will be necessary to investigate this issue in depth.

\section{CONCLUSION}

The main finding of the present study is that $\mathrm{AD}$ patients were able to voluntarily imitate the kinematic features of a moving stimulus, an ability that improved when watching the human demonstrator compared to the computerized object. The present findings may be clinically relevant for cognitive interventions, especially when the efficacy of computer-based techniques is compared with that of traditional training programs, where the therapist plays an active role. These innovative computerbased methodologies have been proposed for cognitive training (Hofmann et al., 1996, 2003), stimulation (Tárraga et al., 2006), and rehabilitation treatments (Cipriani et al., 2006). However, the effectiveness of these techniques, and in particular the improvements they could bring to daily-life activities, is still greatly debated (Bahar-Fuchs et al., 2013). In the present work, by voluntarily imitating the dot velocity, AD patients exhibited their ability to interact with a computerized system, encouraging the use of computerized exercises in cognitive interventions. Nevertheless, the improvement in imitation performance when patients faced the demonstrator suggests that the presence of a human agent could increase the efficacy of the treatment. 


\section{AUTHOR CONTRIBUTIONS}

$\mathrm{AB}$ and $\mathrm{TP}$ conceived and designed the experiments. $\mathrm{AB}, \mathrm{MC}$, $\mathrm{YB}$, and $\mathrm{QW}$ performed the experiments. $\mathrm{AB}, \mathrm{MC}, \mathrm{YB}$ and $\mathrm{TP}$

\section{REFERENCES}

Aron, A. R., Schlaghecken, F., Fletcher, P. C., Bullmore, E. T., Eimer, M., Barker, R., et al. (2003). Inhibition of subliminally primed responses is mediated by the caudate and thalamus: evidence from functional MRI and Huntington's disease. Brain 126, 713-723. doi: 10.1093/brain/awg067

Bahar-Fuchs, A., Clare, L., and Woods, B. (2013). Cognitive training and cognitive rehabilitation for mild to moderate Alzheimer's disease and vascular dementia. Cochrane Database Syst. Rev. 6:CD003260. doi: 10.1002/1465 1858.CD003260.pub2

Bisio, A., Casteran, M., Ballay, Y., Manckoundia, P., Mourey, F., and Pozzo, T. (2012). Motor resonance mechanisms are preserved in Alzheimer's disease patients. Neuroscience 222, 58-68. doi: 10.1016/j.neuroscience.2012.07.017

Bisio, A., Sciutti, A., Nori, F., Metta, G., Fadiga, L., Sandini, G., et al. (2014). Motor contagion during human-human and human- robot interaction. PLoS One 9:e106172. doi: 10.1371/journal.pone.0106172

Bisio, A., Stucchi, N., Jacono, M., Fadiga, L., and Pozzo, T. (2010). Automatic versus voluntary motor imitation: effect of visual context and stimulus velocity. PLoS One 5:e13506. doi: 10.1371/journal.pone.0013506

Brainard, D. H. (1997). The psychophysics toolbox. Spat. Vis. 10, 433-436. doi: 10. $1163 / 156856897 \times 00357$

Brass, M., and Heyes, C. (2005). Imitation: is cognitive neuroscience solving the correspondence problem? Trends Cogn. Sci. 9, 489-495. doi: 10.1016/j.tics. 2005.08.007

Buckner, R. L. (2004). Memory and executive function in aging and ad: multiple factors that cause decline and reserve factors that compensate. Neuron 44, 195-208. doi: 10.1016/j.neuron.2004.09.006

Byrne, R. W., and Russon, A. E. (1998). Learning by imitation: a hierarchical approach. Behav. Brain Sci. 21, 667-684; discussion 684-721. doi: 10. 1017/s0140525x98001745

Chartrand, T. L., and Bargh, J. A. (1999). The chameleon effect: the perceptionbehavior link and social interaction. J. Pers. Soc. Psychol. 76, 893-910. doi: 10. 1037/0022-3514.76.6.893

Cipriani, G., Bianchetti, A., and Trabucchi, M. (2006). Outcomes of a computerbased cognitive rehabilitation program on Alzheimer's disease patients compared with those on patients affected by mild cognitive impairment. Arch. Gerontol. Geriatr. 43, 327-335. doi: 10.1016/j.archger.2005.12.003

Cronin-Golomb, A., Sugiura, R., Corkin, S., and Growdon, J. H. (1993). Incomplete achromatopsia in Alzheimer's disease. Neurobiol. Aging 14, 471-477. doi: 10.1016/0197-4580(93)90105-k

de Gelder, B., de Borst, A. W., and Watson, R. (2014). The perception of emotion in body expressions. Wiley Interdiscip. Rev. Cogn. Sci. 6, 149-158. doi: 10. 1002/wcs. 1335

Edwards, D. F., Baum, C. M., and Deuel, R. K. (1991). Constructional apraxia in Alzheimer's disease: contributions to functional loss. special issue: the mentally impaired elderly: strategies and interventions to maintain function. Phys. Occup. Ther. Geriatr. 9, 53-68. doi: 10.1080/j148v09n03_05

Ghilardi, M. F., Alberoni, M., Marelli, S., Rossi, M., Franceschi, M., Ghez, C., et al. (1999). Impaired movement control in Alzheimer's disease. Neurosci. Lett. 260, 45-48. doi: 10.1016/S0304-3940(98)00957-4

Gilmore, G. C., Wenk, H. E., Naylor, L. A., and Koss, E. (1994). Motion perception and Alzheimer's disease. J. Gerontol. 49, P52-P57. doi: 10.1093/geronj/49. 2.P52

Goldenberg, G. (1999). Matching and imitation of hand finger postures in patients with damage in the left or right hemisphere. Neuropsychologia 37, 559-566. doi: 10.1016/s0028-3932(98)00111-0

Heyes, C. (2011). Automatic imitation. Psychol. Bull. 137, 463-483. doi: 10. 1037/a0022288

Hofmann, M., Hock, C., Kühler, A., and Müller-Spahn, F. (1996). Interactive computer-based cognitive training in patients with Alzheimer's disease. J. Psychiatr. Res. 30, 493-501. doi: 10.1016/s0022-3956(96)00036-2 analyzed the data. $\mathrm{AB}, \mathrm{FM}, \mathrm{PM}$ and $\mathrm{TP}$ interpreted the data. FM, PM and TP contributed the materials. $\mathrm{AB}$ and TP wrote the article. MC, YB, FM, PM and TP revised the article. MC, YB, FM, $\mathrm{PM}$ and TP gave the final approval.

Hofmann, M., Rösler, A., Schwarz, W., Müller-Spahn, F., Kräuchi, K., Hock, C., et al. (2003). Interactive computer-training as a therapeutic tool in Alzheimer's disease. Compr. Psychiatry 44, 213-219. doi: 10.1016/s0010-440x(03) 00006-3

Iacoboni, M., Woods, R. P., Brass, M., Bekkering, H., Mazziotta, J. C., and Rizzolatti, G. (1999). Cortical mechanisms of human imitation. Science 286, 2526-2528. doi: 10.1126/science.286.5449.2526

Korman, J., Voiklis, J., and Malle, B. (2015). The social life of cognition. Cognition 135, 30-35. doi: 10.1016/j.cognition.2014.11.005

Lhermitte, F., Pillon, B., and Serdaru, M. (1986). Human autonomy and the frontal lobes. Part I: imitation and utilization behavior: a neuropsychological study of 75 patients. Ann. Neurol. 19, 326-334. doi: 10.1002/ana.410190404

Meltzoff, A. N., and Moore, M. K. (1977). Imitation of facial and manual gestures by human neonates. Science 198, 74-78. doi: 10.1126/science. 897687

Mendez, M. F., Chen, A. K., Shapira, J. S., and Miller, B. L. (2005). Acquired sociopathy and frontotemporal dementia. Dement. Geriatr. Cogn. Disord. 20, 99-104. doi: 10.1159/000086474

Narme, P., Mouras, H., Roussel, M., Devendeville, A., and Godefroy, O. (2013). Assessment of socioemotional processes facilitates the distinction between frontotemporal lobar degeneration and Alzheimer's disease. J. Clin. Exp. Neuropsychol. 35, 728-744. doi: 10.1080/13803395.2013.823911

Nithianantharajah, J., and Hannan, A. J. (2009). The neurobiology of brain and cognitive reserve: mental and physical activity as modulators of brain disorders. Prog. Neurobiol. 89, 369-382. doi: 10.1016/j.pneurobio.2009. 10.001

Pandey, S., and Sarma, N. (2015). Utilization behavior. Ann. Indian Acad. Neurol. 18, 235-237. doi: 10.4103/0972-2327.150613

Perry, R. J., and Hodges, J. R. (1999). Attention and executive deficits in Alzheimer's disease. A critical review. Brain 122, 383-404. doi: 10. 1093/brain/122.3.383

Rankin, K. P., Kramer, J. H., Mychack, P., and Miller, B. L. (2003). Double dissociation of social functioning in frontotemporal dementia. Neurology 60, 266-271. doi: 10.1212/01.wnl.0000041497.07694.d2

Rankin, K. P., Santos-Modesitt, W., Kramer, J. H., Pavlic, D., Beckman, V., and Miller, B. L. (2008). Spontaneous social behaviors discriminate behavioral dementias from psychiatric disorders and other dementias. J. Clin. Psychiatry 69, 60-73. doi: 10.4088/jcp.v69n0109

Rapcsak, S. Z., Croswell, S. C., and Rubens, A. B. (1989). Apraxia in Alzheimer's disease. Neurology 39, 664-668. doi: 10.1212/WNL.39.5.664

Rizzo, M., and Nawrot, M. (1998). Perception of movement and shape in Alzheimer's disease. Brain 121, 2259-2270. doi: 10.1093/brain/121.12. 2259

Rizzolatti, G., Fadiga, L., Fogassi, L., and Gallese, V. (1999). Resonance behaviors and mirror neurons. Arch. Ital. Biol. 137, 85-100.

Rosen, H., Narvaez, J., Hallam, B., Kramer, J., Wyss-Coray, C., Gearhart, R., et al. (2004). Neuropsychological and functional measures of severity in Alzheimer disease, frontotemporal dementia and semantic dementia. Alzheimer Dis. Assoc. Disord. 18, 202-207.

Rousseaux, M., Rénier, J., Anicet, L., Pasquier, F., and Mackowiak-Cordoliani, M. A. (2012). Gesture comprehension, knowledge and production in Alzheimer's disease. Eur. J. Neurol. 19, 1037-1044. doi: 10.1111/j.1468-1331. 2012.03674.x

Shany-Ur, T., and Rankin, K. P. (2011). Personality and social cognition in neurodegenerative disease. Curr. Opin. Neurol. 24, 550-555. doi: 10. 1097/WCO.0b013e32834cd42a

Tárraga, L., Boada, M., Modinos, G., Espinosa, A., Diego, S., Morera, A., et al. (2006). A randomised pilot study to assess the efficacy of an interactive, multimedia tool of cognitive stimulation in Alzheimer's disease. J. Neurol. Neurosurg. Psychiatry 77, 1116-1121. doi: 10.1136/jnnp.2005. 086074 
Tippett, W. J., Krajewski, A., and Sergio, L. E. (2007). Visuomotor integration is compromised in Alzheimer's disease patients reaching for remembered targets. Eur. Neurol. 58, 1-11. doi: 10.1159/000102160

Tippett, W. J., and Sergio, L. E. (2006). Visuomotor integration is impaired in early stage Alzheimer's disease. Brain Res. 1102, 92-102. doi: 10.1016/j.brainres. 2006.04.049

Travniczek-Marterer, A., Danielczyk, W., Simanyi, M., and Fischer, P. (1993). Ideomotor apraxia in Alzheimer's disease. Acta Neurol. Scand. 88, 1-4. doi: 10. 1111/j.1600-0404.1993.tb04176.x

Vallet, G. T. (2015). Embodied cognition of aging. Front. Psychol. 6:463. doi: 10. 3389/fpsyg.2015.00463
Conflict of Interest Statement: The authors declare that the research was conducted in the absence of any commercial or financial relationships that could be construed as a potential conflict of interest.

Copyright (C) 2016 Bisio, Casteran, Ballay, Manckoundia, Mourey and Pozzo. This is an open-access article distributed under the terms of the Creative Commons Attribution License (CC BY). The use, distribution and reproduction in other forums is permitted, provided the original author(s) or licensor are credited and that the original publication in this journal is cited, in accordance with accepted academic practice. No use, distribution or reproduction is permitted which does not comply with these terms. 